\title{
Modelos fantásticos: los secretos del software dinámico
}

\section{Fantastic models: the secrets of dynamic software}

DOI: $10.54019 /$ sesv3n1-025

Recebimento dos originais: $15 / 01 / 2022$

Aceitação para publicação: 10/02/2022

\begin{abstract}
Aitzol Lasa
Doctor en Didáctica de las Matemáticas

Universidad Pública de Navarra (UPNA)

E-mail: aitzol.lasa@unavarra.es

Miguel R. Wilhelmi

Doctor en Didáctica de las Matemáticas

Universidad Pública de Navarra (UPNA)

E-mail: miguelr.wilhelmi@unavarra.es
\end{abstract}

\section{Jaione Abaurrea}

Master en Profesorado en Educación Secundaria

Universidad Pública de Navarra (UPNA)

E-mail: jaione.abaurrea@unavarra.es

\section{RESUMEN}

El Grupo de investigación en Didáctica de las matemáticas de la UPNA trabaja desde 2009 en el diseño de modelos dinámicos y en su implementación en situaciones de enseñanza y aprendizaje de las matemáticas. En este artículo, se muestra una galería de estos materiales, que van desde la Educación Infantil hasta la Universidad. Cada modelo se acompaña con sus respectivas explicaciones y justificaciones teóricas, que se fundamentan en un modelo didáctico basado en tres teorías: Teoría de situaciones didácticas en matemáticas; Enfoque ontosemiótico del conocimiento y la instrucción matemáticos; y Teoría de la génesis instrumental. El Grupo de investigación diseña y analiza sus propuestas con la metodología de la Ingeniería didáctica basada en el enfoque ontosemiótico. Las experiencias realizadas validan el uso de los modelos dinámicos para la implementación de situaciones de enseñanza y aprendizaje de las matemáticas. El software de geometría dinámica es pues un instrumento eficaz, y la clasificación de modelos en términos de exploración, ilustración y demostración explica las tipologías de la actividad matemática que se puede realizar con software dinámico. 
Palabras clave: GeoGebra (GGB), Teoría de situaciones didácticas en matemáticas (TSDM), Teoría de la génesis instrumental (TGI), Enfoque ontosemiótico (EOS).

\section{ABSTRACT}

The Didactics of Mathematics Research Group at UPNA has been working since 2009 on the design of dynamic models and their implementation in mathematics teaching and learning situations. In this article, we show a gallery of these materials, ranging from Early Childhood Education to University. Each model is accompanied by its respective explanations and theoretical justifications, which are based on a didactic model based on three theories: Theory of didactic situations in mathematics; Ontosemiotic approach to mathematical knowledge and instruction; and Theory of instrumental genesis. The research group designs and analyzes its proposals with the didactic engineering methodology based on the ontosemiotic approach. The experiences carried out validate the use of dynamic models for the implementation of mathematics teaching and learning situations. Dynamic geometry software is thus an effective tool, and the classification of models in terms of exploration, illustration and demonstration explains the typologies of mathematical activity that can be performed with dynamic software.

Keywords: GeoGebra (GGB), Theory of didactic situations in mathematics (TSDM), Theory of instrumental genesis (TGI), Ontosemiotic approach (EOS).

\section{INTRODUCCIÓN}

Si bien el software de geometría dinámica tiene un recorrido de varias décadas, la programación informática del software y el diseño de su interface deben estar justificadas y abaladas por una teoría didáctica. El punto de partida de esta base didáctica se fundamenta dentro de la Teoría de la educación matemática realista (Freudenthal, 1978) y se complementa con el Aprendizaje a través de modelos (de Jong, van Joolingen, 2008; Milrad, M., Spector, J.M., Davidsen, 2003), que incorporan además desarrollos más recientes en el uso de tecnología en la educación matemática, tales como la Teoría de la génesis instrumental (TGI).

En este contexto, el Grupo de investigación en didáctica de las matemáticas de la UPNA (figura 1) trabaja desde 2009 en el diseño de modelos dinámicos y en su implementación en situaciones de enseñanza y aprendizaje de las matemáticas. En este tiempo, se ha avanzado desde la descripción del uso de GeoGebra como herramienta en contextos de formación inicial de profesorado 
(Lasa, Sáenz de Cabezón y Wilhelmi, 2009) y su conexión con planes de formación (objetivos de aprendizaje, procedimientos de asesoría, presentación de actividades y ejemplos) a un modelo teórico que integra la instrumentación con GeoGebra en procesos de enseñanza y aprendizaje.

Figura 1. Logotipos del Grupo de investigación DIDAMATIK y UPNA.

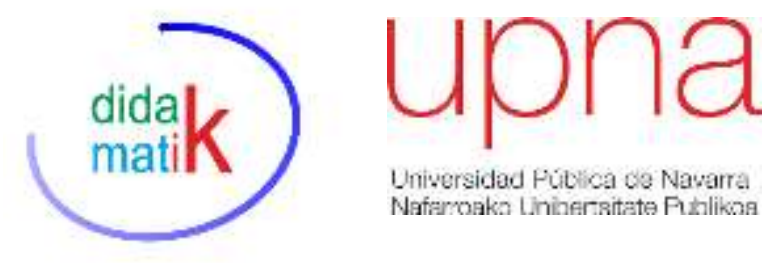

GeoGebra permite al docente una gestión del aula y un control del proceso de enseñanza y aprendizaje centrada en propiedades y no en ejemplos particulares. A las motivaciones puramente pragmáticas (idiomáticas, técnicas y económicas) hay que añadir motivaciones epistemológicas y educativas: presenta herramientas para el desarrollo integrado de nociones, procesos y significados propios de la geometría, el álgebra, la teoría de funciones, la estadística y la probabilidad, que permiten resaltar el aspecto esencialmente relacional de las matemáticas.

El uso de la pizarra digital complementa la visualización de contenidos mediante GeoGebra, ya que el uso de la pizarra clásica tiene la limitación evidente de no poder mostrar más que un ejemplo, o de unos pocos, que pueden generar un fenómeno de ilusión de la transparencia (Lasa, Wilhelmi, 2013a). GeoGebra contribuye a superar este fenómeno, acortando la distancia entre la interpretación de un ejemplo en tanto "objeto aislado" y en tanto "representante de una clase".

En este artículo, se muestra una galería de estos materiales, que van desde la Educación Infantil hasta la Universidad. Cada modelo se acompaña con sus respectivas explicaciones y justificaciones teóricas, y son accesibles vía código QR; para ello, basta con fotografiar el código con un teléfono móvil. 


\section{MODELO DIDÁCTICO Y METODOLÓGICO}

El diseño de los modelos y la implementación de las situaciones en las que estos se utilizan siguen un modelo didáctico que se basa fundamentalmente en tres teorías: La Teoría de situaciones didáctica en matemáticas (TSDM, Brousseau); la Teoría de la génesis instrumental (TGI, Rabardel); y el Enfoque ontosemiótico del conocimiento y la instrucción matemáticos (EOS, Godino).

Según la TSDM (Brousseau, 2007), todo conocimiento matemático se puede modelizar mediante una situación de aprendizaje, denominada por ello fundamental. Los estudiantes que se enfrentan a esta situación, la cual tiene un componente adidáctico esencial (Bloch, 1999), adquieren dicho conocimiento matemático siguiendo unas pautas marcadas en fases de acción, formulación y validación.

Los estudiantes aportan respuestas adaptadas a la situación, y estas respuestas son muestra de su conocimiento personal y funcional de las matemáticas. Si la situación varía, bien porque se ha cambiado el objetivo de la tarea, el contenido matemático, el medio material, etc., los estudiantes tratan de adaptar su conocimiento previo para dar respuesta a la nueva situación. Un conocimiento estable puede generar un obstáculo de tipo epistemológico, si resiste a las contradicciones a las que se somete, provocando errores específicos, localizables, analizables y recurrentes, que se siguen manifestando en el tiempo y en diversos contextos.

Por ello, la adquisición de nuevo conocimiento requiere una programación sistemática, por parte del docente o del investigador, de actividades y situaciones de aprendizaje a las que el estudiante se debe enfrentar, que termina por adaptar o sustituir el conocimiento previo que había generado, de manera que el estudiante termina por superar el obstáculo.

Las intervenciones específicas que realizan el docente o el investigador, contemplan el particular un cambio de registro y el uso de diversos medios materiales. Según la TGI (Drijvers, Godino, Font y Trouche, 2013), una herramienta o artefacto es un objeto que se utiliza para desempeñar una tarea y que tradicionalmente se ha identificado con materiales manipulativos y que ahora 
se asocia en muchas ocasiones con un soporte digital. La herramienta se convierte en instrumento cuando la persona que lo manipula lo utiliza en la resolución de una tarea determinada, aportando ciertas destrezas necesarias en su resolución.

Los obstáculos se pueden superar, precisamente, mediante un esquema de acción instrumentada (Trouche, 2000), que implica, por parte de los estudiantes, una forma estable de resolver una tarea específica mediante un instrumento determinado. Estos instrumentos bien pueden ser un soporte físico y manipulable (bloques, puzles, palillos, cubos, etc.), el soporte de lápiz y papel (un papel blanco, un papel cuadriculado, una plantilla donde aplicar instrumentos de dibujo, etc.), o un software (hoja de cálculo, geometría dinámica, etc.).

El soporte material utilizado condiciona el significado que el estudiante atribuye al objeto matemático de estudio, y por ello, la integración de diversos soportes ayuda al estudiante en la comprensión del contenido matemático. Es decir, la capacidad de modificación de los registros semióticos mejora el aprendizaje del estudiante (Duval, 1995).

EI EOS (Font, Godino, y D’Amore, 2007; Font, 2007) también entiende que, tanto la instrumentación como la instrumentalización, suponen una práctica operativa y discursiva determinada, motivada por una familia de situaciones, y que requiere de un lenguaje propio, de una serie de definiciones, de enunciados y de argumentos. Es precisamente la manipulación de objetos matemáticos la que define la actividad matemática, y estos objetos matemáticos pueden tener múltiples representaciones. La realidad contextual de los objetos matemáticos se concreta en su funcionamiento atendiendo a distintas dualidades: extensivos (particulares) e intensivos (genéricos); significado personal (cognitivo) e institucional (epistemológico); carácter ostensivo (material) y no ostensivo (ideal); unitario y sistémico; y de contenido y expresión.

Así pues, el diseño de una situación didáctica tiene el propósito de institucionalizar el significado de referencia de un contenido matemático, y para ello, articula una situación en términos de fases de acción, formulación y validación, en la cual los estudiantes ponen en funcionamiento el significado 
personal del contenido matemático para resolver la tarea propuesta. Los objetos matemáticos ideales que el estudiante debe adquirir, se materializan sobre una variedad de instrumentos, en contraposición al significado ideal de estos mismos objetos.

La ventaja de la utilización de un modelo dinámico como soporte de la actividad matemática es que pone el foco de atención sobre las propiedades generales de los objetos matemáticos involucrados, a la vez que facilita los medios para analizar un gran número de casos particulares. La instrumentación del soporte material utilizado no es la única práctica operativa que determina la actividad matemática escolar, puesto que esta actividad requiere de la utilización del número y de representaciones algebraicas en sus distintos niveles.

Además, el software de geometría dinámica posibilita la progresión de los estudiantes en la adquisición de los sucesivos niveles de comprensión y utilización del lenguaje algebraico, que van desde una utilización puramente aritmética en ausencia de lenguaje algebraico (nivel 1 de algebrización), hasta un nivel consolidado (nivel3), empezando desde edades tempranas (Godino, Castro, Aké y Wilhelmi, 2012; Godino, Aké, Gonzato y Wilhelmi, 2014).

En resumen, la TSDM y la TGI, junto con el EOS, permiten diseñar propuestas de enseñanza y aprendizaje, y analizar los resultados obtenidos en estas experiencias. El equipo de investigación diseña y analiza sus propuestas con la metodología de la Ingeniería didáctica basada en el enfoque ontosemiótico (Godino, Rivas, Arteaga, Lasa, Wilhelmi, 2014).

\section{CLASIFICACIÓN Y EJEMPLIFICACIÓN DE MODELOS}

Se consideran tres momentos fundamentales en los que el uso de GGB es pertinente en la actividad matemática escolar, y los recursos empleados se clasifican, según esta utilización, en modelos de exploración, ilustración, o demostración (Lasa, Wilhelmi, 2013b).

El software de geometría dinámica permite la construcción de modelos exploratorios para resolver ejercicios y problemas. Normalmente, estas construcciones no las realiza el propio estudiante, las diseña el docente o éste las 
selecciona desde un catálogo de recursos. El objetivo es diseñar una construcción que satisfaga las restricciones de una proposición o las condiciones iniciales de un problema. Después de manipular la construcción, los estudiantes deducen sus propiedades. En los modelos de exploración, la construcción cumple las condiciones iniciales de un problema, y el estudiante explora la situación para extraer conclusiones e inferir propiedades desconocidas hasta el momento; obtiene el feedback de la construcción.

El uso generalizado de GGB consiste en presentar construcciones que muestran la veracidad de una propiedad, que deben motivar a su vez nuevos ejemplos que mejoren la confianza de los estudiantes en las conjeturas formuladas. Hölz (2001), citado por Burke y Kennedy (2011), afirma que, en un entorno de software de geometría dinámica, el estudiante que observa la verdad de una conjetura siente la necesidad de conocer la razón que justifica esta afirmación. Después de todo, la ilustración de una propiedad es solo una "imagen" de ella. En la Educación Secundaria, las restricciones materiales y temporales, o las restricciones cognitivas de los estudiantes pueden llevar a la decisión didáctica de ignorar la demostración formal de la propiedad, limitando la actividad a una presentación ilustrativa de la misma. En un modelo de ilustración, la construcción cumple las restricciones de una proposición, y el estudiante intenta encontrar un contraejemplo sobre una multitud de casos particulares; al no poder contradecir la proposición la da por buena, basándose en argumentos inductivos. Sin embargo, puede haber divergencias entre la construcción ilustrativa de una propiedad y la prueba formal de esta misma propiedad.

Tradicionalmente, la prueba formal de una propiedad geométrica se lleva a cabo paso a paso en una pizarra. Sin embargo, a medida que las pizarras ordinarias son sustituidas por pizarras digitales y software de geometría dinámica, estas pruebas formales se dejan de lado y se sustituyen por construcciones ilustrativas, que a veces no se diseñan teniendo en cuenta elementos de la prueba formal, y entonces, los pasos informáticos de la construcción difieren del razonamiento lógico formal. Es tarea del maestro seleccionar situaciones que permitan unir ambos razonamientos; el razonamiento inductivo debido al software 
de geometría dinámica, y el razonamiento deductivo, tradicionalmente vinculado a pruebas formales (pruebas de lápiz y papel). Por ello, en este momento, el docente selecciona situaciones que permitan al estudiante complementar razonamientos inductivos y deductivos, utilizando varios soportes (software, y lápiz y papel), utilizando modelos de demostración.

\section{GALERÍA DE MODELOS DINÁMICOS}

Se presentan a continuación las descripciones de una serie de construcciones dinámicas junto con los enlaces a estos mismos recursos. Con el propósito de facilitar el acceso a los recursos, se incorpora un código QR que permite el acceso directo mediante una fotografía del mismo. Los recursos abarcan las etapas educativas de Educación Infantil (de 3 a 5 años) y Primaria (de 6 a 11 años), la Educación Secundaria Obligatoria (de 12 a 15 años) y el Bachillerato (de 16 a 17 años) y Universidad (a partir de 18 años). Estas referencias de etapas y edades corresponden al sistema educativo en España.

\subsection{EDUCACIÓN INFANTIL Y PRIMARIA}

En las primeras etapas del sistema educativo, los estudiantes adquieren paulatinamente el léxico formal de la geometría plana. El significado de los términos punto, segmento, recta, etc., se puede abordar mediante el medio material GGB, con un formato de Libro-GeoGebra, es decir, con un recurso digital que recopila una serie de actividades con una estructura similar, que facilita al estudiante la familiarización con el medio, y que se realizan de manera secuencial.

El primer recurso (Lasa, 2016) se denomina Puntos móviles en el plano (figura 2). En él, el estudiante pulsa y arrastra sobre el punto de color azul, el cual se ha programado previamente para que realice un itinerario determinado (punto libre, punto sobre un segmento, una recta, un polígono, etc.). Tras este momento de acción, el estudiante formula su conjetura, que podrá validar pulsando sobre los botones "muestra rastro" (ahora, el movimiento del punto azul dejará un rastro tras de sí, dibujando la figura) o "muestra rótulo" (se abre una ventana de texto que indica el nombre de la figura). 
Figura 2. Interface, enlace y código QR del recurso "Puntos Móviles en el Plano".

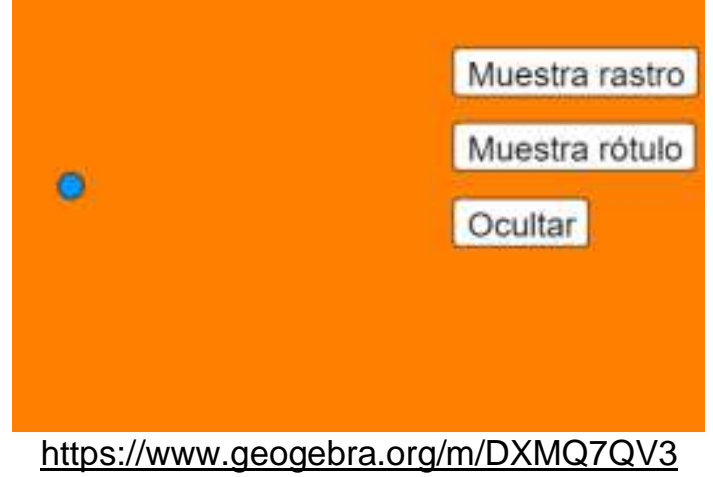

Muestra rastro

Muestra rótulo

Ocultar

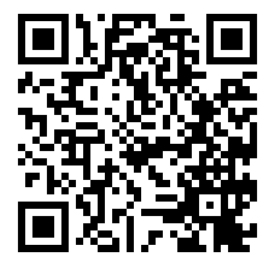

La actividad matemática en las primeras etapas educativas es fundamentalmente verbal, y por ello, no suele aparecer aislada del aprendizaje del lenguaje. En el recurso Larraintzar (Lasa, 2015) se trabaja el aprendizaje de la simetría plana de eje vertical, mediante actividades de análisis de figuras geométricas simétricas y la lectura de las letras del alfabeto con eje de simetría vertical (figura 3). En particular, la actividad se centra en el estudio de la palabra AMATXO, "madrecita" en vasco, por ser una palabra hacia la que los estudiantes de edades tempranas sienten una importante vinculación afectiva, y cuyas letras contienen un eje vertical de simetría.

La capacidad de atención de los estudiantes en las edades tempranas es limitada, por ello, el recurso contiene una serie de puzles y animaciones que intercalan actividades guiadas con actividades artísticas en formato libre. En todas las animaciones, las letras con simetría vertical contienen movimiento, mientras que las demás letras permanecen estáticas. En las actividades guiadas, el estudiante debe completar figuras simétricas mediante arrastre, composición y descomposición de figuras planas. En las actividades libres, el recurso está programado a modo de caleidoscopio, de manera que cuando el estudiante dibuja de manera libre una parte de la imagen, el software completa la figura mediante una simetría de eje vertical. 
Figura 3. Interface, enlace y código QR del recurso "Larraintzar".
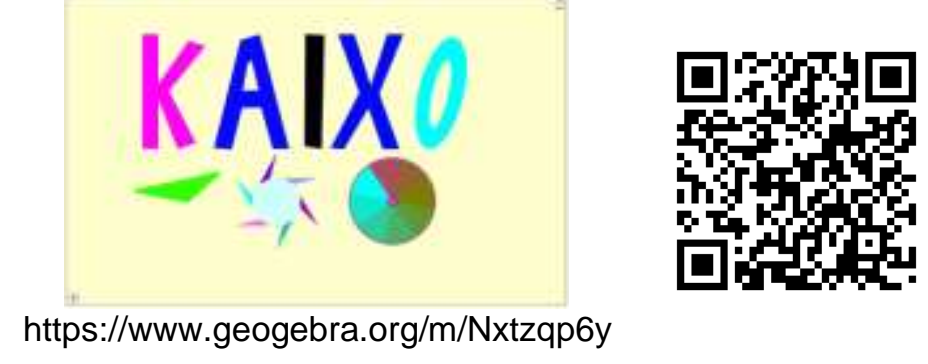

https://www.geogebra.org/m/Nxtzqp6y

A medida que los estudiantes avanzan en el sistema educativo, se incorporan nuevos contenidos geométricos, así como las nociones de medida de magnitudes lineales y planas. En la situación didáctica "Una parcela para Txuri" (figura 4), se introduce la optimización de áreas en segundo ciclo de Educación Primaria (8-9 años), mediante una propuesta que articula la utilización de dos soportes físicos: uno, lápiz y papel; otro, software de geometría dinámica (Lasa, Wilhelmi, Belletich, 2014). Se introduce un modelo dinámico en un momento de exploración, una vez que la actividad matemática sobre papel excede el grado de maestría aritmética de los niños.

La consigna indica que la perra Txuri "necesita espacio para jugar con su pelota, pero si se queda suelta, se despista y no sabe volver. Por ello, queremos construir una parcela para Txuri, en la cual pueda jugar. Disponemos de 20 metros de valla, y con esos 20 metros de perímetro, tenemos que construir un cerco que cubra el mayor espacio posible". Los estudiantes emplean en el análisis de la situación hasta tres soportes materiales: material físico y manipulable (palillos, cuerda, etc.), un soporte de papel cuadriculado sobre el que dibujan formas geométricas planas, y un modelo dinámico de exploración.

El desarrollo de la actividad ejemplifica la manera en la que cada uno de estos soportes influye en las respuestas de los estudiantes. La instrumentación del modelo dinámico permite a los estudiantes identificar la circunferencia como la figura plana que optimiza el área para un perímetro determinado, a la vez que los estudiantes aportan una variedad de ejemplos que certifican la propiedad isoperimétrica, dotando de significado a los términos perímetro y área, y 
superando el obstáculo didáctico asociado a su identificación como un mismo objeto geométrico.

Figura 4. Interface, enlace y código QR del recurso "Una parcela para Txuri / A plot for Laika".

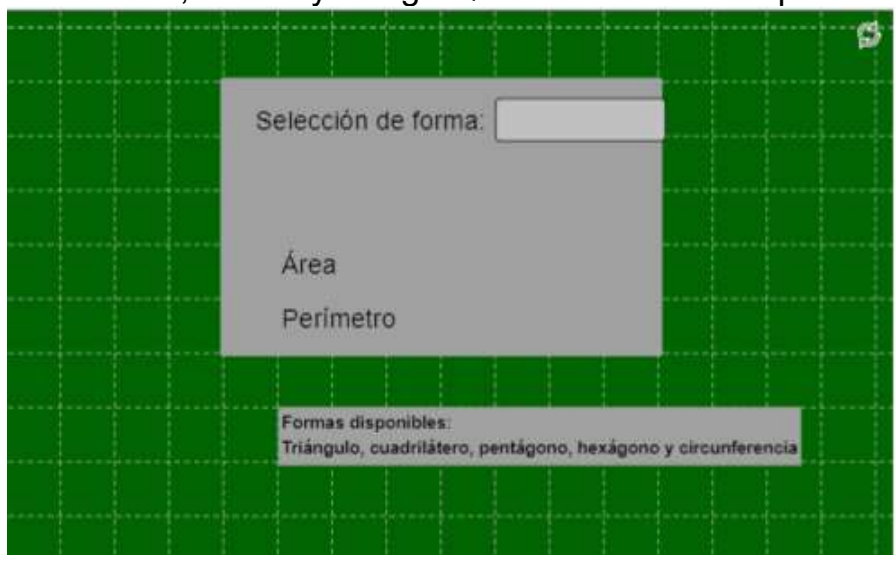

https://www.geogebra.org/m/FjJDGxuK

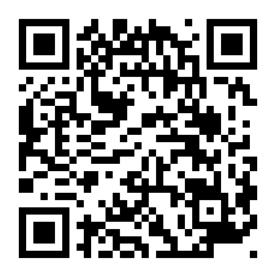

\subsection{EDUCACIÓN SECUNDARIA OBLIGATORIA Y BACHILLERATO}

La etapa post-elemental abre la puerta a nuevos contenidos matemáticos, y los estudiantes avanzan asimismo en la adquisición de nuevo lenguaje algebraico. En la actividad matemática escolar, el software cumple un objetivo claro en relación al uso de operaciones algebraicas: siempre que la actividad matemática propuesta al estudiante exceda el umbral de maestría aritmética del mismo, el software desbloquea la situación, permitiendo al estudiante avanzar en la comprensión de nuevos contenidos.

El siguiente ejemplo lleva por título Elogio de los triángulos, y se trata de un Libro-GeoGebra para asistir situaciones de enseñanza y aprendizaje de la trigonometría (Lasa, Abaurrea, Belloso, 2016). En este recurso, se emplean modelos de exploración, ilustración y demostración, para avanzar en la comprensión de las nociones fundamentales de ratio trigonométrico, la transición desde esta noción básica a la noción de función trigonométrica, y se finaliza con un estudio de la función de honda y diversas propiedades trigonométricas de la misma. 
Figura 5. Interface, enlace y código QR del recurso "Elogio de los triángulos".

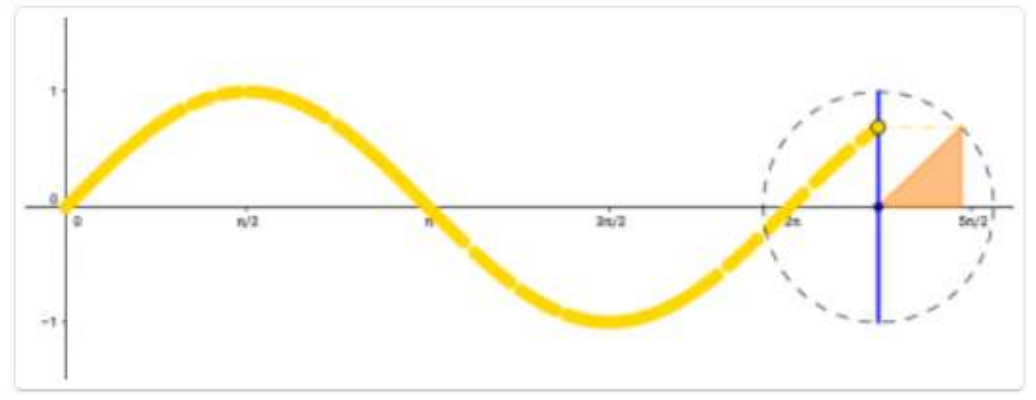

https://www.geogebra.org/m/d2exF80t

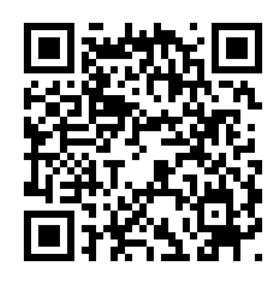

Otro ejemplo es el modelo dinámico que permite aplicar el Método del cuadrado (Abaurrea, Lasa, Wilhelmi, 2019) para la determinar y representar una circunferencia partiendo de su representación algebraica no canónica (figura 6). Wilhelmi (2007) propone cuatro métodos analíticos para la determinación de circunferencias. Tres de ellos requieren de un análisis algebraico previo que determine el número de puntos de corte de la circunferencia con los ejes de coordenadas; ya que, en base a esta información, se debe aplicar un método u otro. Sin embargo, el Método del cuadrado es aplicable para representar cualquier circunferencia, independientemente de la cantidad de puntos de corte.

Su aplicabilidad a todas las circunferencias hace que el Método del cuadrado resulte interesante como objeto de estudio. Este método de representación difiere del mostrado habitualmente en los libros de texto o recursos didácticos; que suele centrarse en la representación de la circunferencia partiendo siempre de su ecuación en forma canónica que permite a los estudiantes obtener información sobre el centro y radio de la curva. En cambio, el Método del cuadrado consiste en determinar un cuadrado que delimita la circunferencia.

Los estudiantes emplean dos soportes materiales: el soporte "lápiz y papel” y el modelo dinámico de exploración. En primer lugar, deben calcular los valores de " $x$ " e " $y$ " que determinan el cuadrado delimitador tal y como se describe en el modelo dinámico. Una vez conocen los valores máximo y mínimo que alcanzan los puntos de la circunferencia tanto en el eje OX como en el eje OY, éstos se deben introducir en el modelo dinámico para que éste muestre el cuadrado 
delimitador. Llegados a este punto, son los alumnos los que estudian y exploran cuál es la circunferencia inscrita en dicho cuadrado.

Figura 6. Interface, enlace y código QR del recurso "Determinación de una circunferencia".
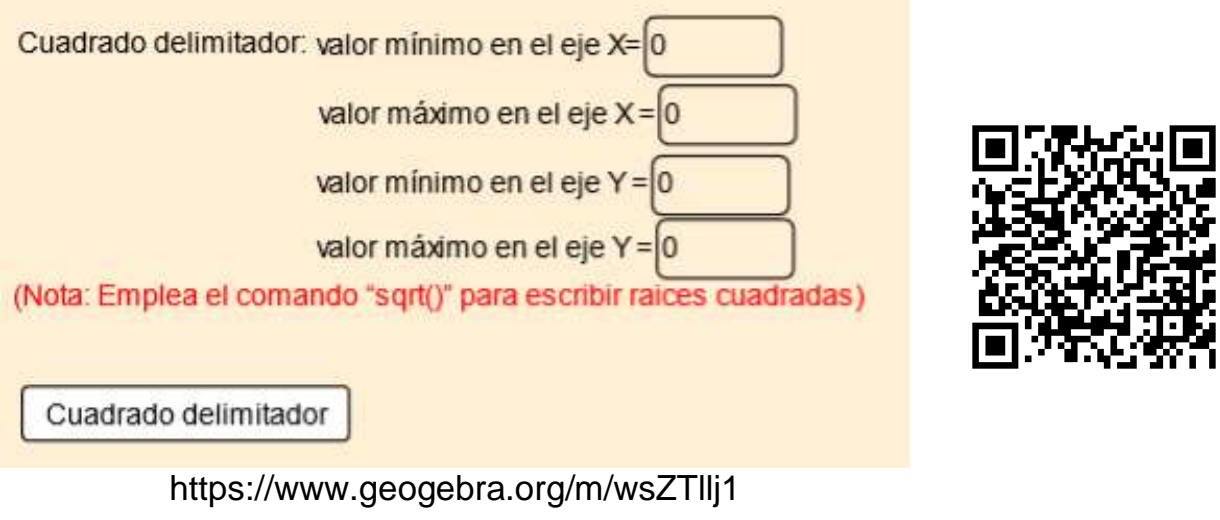

Los modelos de ilustración transcienden el contexto puramente académico o escolar, y facilitan también la divulgación de un contenido científico con trasfondo histórico. El recurso denominado La conferencia perdida de Feynman (Lasa, Wilhelmi, 2015) ejemplifica el uso ilustrativo de un Libro-GeoGebra. En él, se toma punto de partida una anécdota histórica sobre el célebre físico Richard Feynman, famoso entre otras muchas cosas por su habilidad para dar conferencias científicas. Se dice de él que tenía la capacidad de adecuar sus explicaciones al nivel de maestría en física que tuvieran los participantes de la sala. A su vez, tenía fama de mostrar un gran entusiasmo en sus explicaciones.

En el Instituto Caltech donde Feynman impartía sus clases e investigaba, sus compañeros tenían la sana costumbre de grabar en magnetofón estas conferencias, para poder así transcribirlas en papel y poder utilizarlas a modo de texto, casi literal, con sus estudiantes de grado en física. Como apoyo a la grabación, se tomaban también fotografías de la pizarra donde Feynman desarrollaba sus diagramas. Sin embargo, las fotografías analógicas no siempre se han conservado correctamente. Tampoco se han conseguido guardar los apuntes y las anotaciones de las conferencias que él hacía a mano antes de cada sesión. 
En este recurso, se hace la reconstrucción mediante modelos dinámicos ilustrativos, de una conferencia de Feynman sobre el movimiento de los planetas en el sistema solar, de la cual solo se ha conservado la transcripción escrita de su exposición oral. La pregunta que surge a modo de divertimento es sencilla y natural: ¿y si Feynman hubiera tenido a mano software de geometría dinámica para desarrollar sus conferencias? La respuesta a esta pregunta se encuentra en el recurso La conferencia perdida de Feynman.

Figura 7. Interface, enlace y código QR del recurso "La conferencia perdida de Feynman".
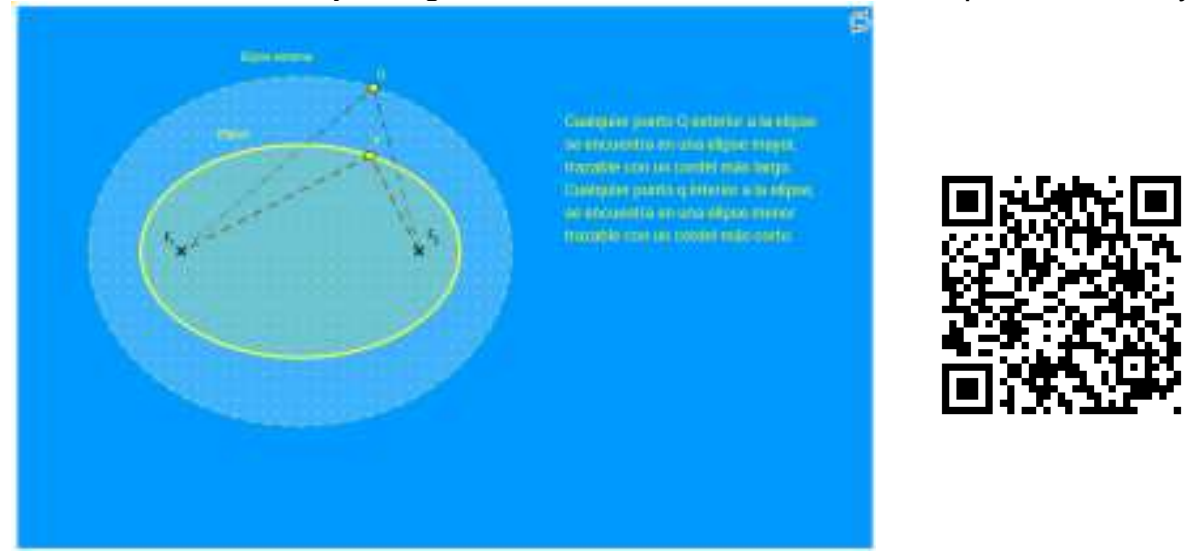

https://www.geogebra.org/m/wfjjKtBa

\subsection{UNIVERSIDAD E INVESTIGACIÓN}

En el marco del Enfoque ontosemiótico del conocimiento y la instrucción matemáticos (EOS) se caracterizan los distintos niveles de adquisición del razonamiento algebraico, desde la Educación Primaria hasta los niveles superiores (Godino, Neto, Wilhelmi, Aké, Etchegaray y Lasa, 2015). Los primeros tres niveles definen los procesos de generalización que implican el cálculo analítico en función de la actividad matemática correspondiente en la Educación Primaria. A continuación, el modelo se amplía mediante la inclusión de otros tres niveles avanzados que permiten la descripción y el análisis de la actividad matemática en la Educación Secundaria, que consideran la utilización de parámetros y estructuras algebraicas.

La descripción de los niveles tiene en cuenta los tipos de representaciones que se emplean, así como la influencia del medio material empleado. El uso de 
dispositivos de cálculo simbólico y representación gráfica como, por ejemplo, GeoGebra, permite manipular con facilidad expresiones algebraicas en las que intervienen variables y parámetros, y, por tanto, familias de ecuaciones y funciones. Las conversiones entre los registros de representación semiótica tabular, gráfica y simbólica de los objetos algebraicos pueden suponer una ayuda en las primeras fases del proceso de reificación de tales objetos.

Se presenta, a modo de ejemplo, un recurso (figura 8) que articula los marcos numérico, simbólico y gráfico mediante GGB. En particular, la herramienta "deslizador" permite la manipulación de coeficientes de funciones afines, es decir, parámetros de familias de funciones.

Figura 8. Interface y código QR del recurso Nivel algebraico avanzado.

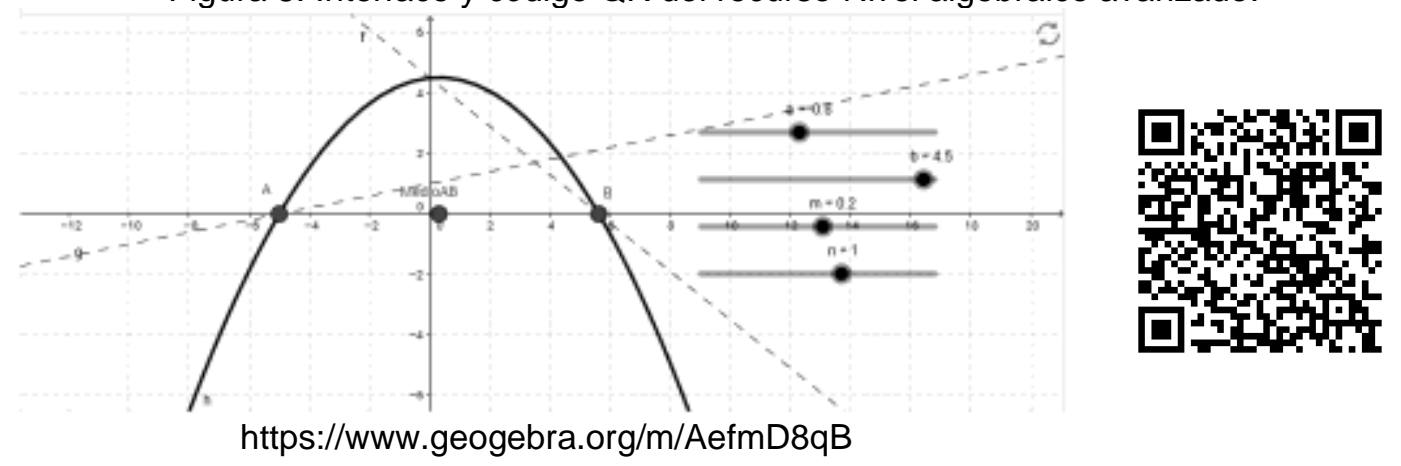

\section{CONCLUSIONES}

El catálogo que aquí se presenta engloba toda una galería de experiencias que, una vez realizadas y estudiadas, permiten validar el uso de los modelos dinámicos para la implementación de situaciones de enseñanza y aprendizaje de las matemáticas, en todas las etapas educativas, desde la Educación Infantil hasta la Universidad. El software de geometría dinámica es pues un instrumento eficaz, que, si bien carece todavía a día de hoy de una presencia hegemónica en las propuestas de enseñanza y aprendizaje de las matemáticas, tampoco muestra síntomas de agotamiento o superación por otros medios materiales o tecnológicos novedosos.

La clasificación de modelos en términos de exploración, ilustración y demostración explica las tipologías de la actividad matemática que se puede 
realizar con software dinámico. Asimismo, la noción de umbral de maestría aritmética de los estudiantes facilita al docente y al investigador la identificación de aquellos hitos o momentos del aprendizaje del estudiante, en los que la actividad matemática escolar puede avanzar gracias a un modelo dinámico, aun cuando el estudiante no tenga un nivel de algebrización acorde con los requerimientos de la actividad, sin que este nivel de algebrización suponga un impedimento o una barrera a la actividad matemática escolar. 


\section{REFERENCIAS}

Abaurrea, J., Lasa, A. y Wilhelmi, M.R. (2019). Momentos de exploración e ilustración en la determinación de circunferencias en futuros docentes de Educación Secundaria. Yupana, 87-104, https://doi.org/10.14409/yu.v0i11.8844

Bloch, I. (1999). L'articulation du travail mathématique du professeur et de l'élève dans l'enseignement de l'analyse en première scientifique. Détermination d'un milieu. Connaissances et savoirs. Recherche en Didactique des Mathématiques, 19(2), 135-194, https://revue-rdm.com/1999/l-articulation-du-travail

Brousseau, G. (2007). Iniciación al estudio de la Teoría de Situaciones Didácticas. Buenos Aires: Zorzal.

Drijvers, P., Godino, J.D., Font, V., Trouche, L. (2013). One episode, two lenses. Educational Studies in Mathematics, 82(1), 23-49, https://doi.org/10.1007/s10649012-9416-8

Duval, R. 1995. Sémiosis et pensée humaine. Registres sémiotiques et apprentissages intellectuels. Berne: Peter Lang.

Font, V. (2007). Una perspectiva ontosemiótica sobre cuatro instrumentos de conocimiento que comparten un aire de familia: particular/general, representación, metáfora y contexto. Educación matemática, 19(2), 95-128. http://www.scielo.org.mx/pdf/ed/v19n2/1665-5826-ed-19-02-95.pdf

Font, V., Godino, J.D., D’Amore, B. (2007). Enfoque ontosemiótico de las representaciones en educación matemática. For the learning of mathematics, 27(2), 2-7, 2ttps://www.ugr.es/ jgodino/funcionessemioticas/sintesis_eos_10marzo08.pdf

Freudenthal, H. (1978). Weeding and sowing: Preface to a science of mathematics education. Dordrecht, Holland: D. Reidel.

Godino, J. D., Aké, L., Gonzato, M., Wilhelmi, M. R. (2014). Niveles de algebrización de la actividad matemática escolar. Implicaciones para la formación de maestros. Enseñanza de las Ciencias, 32(1), 199-219, https://doi.org/10.5565/rev/ensciencias.965

Godino, J. D., Castro, W.F., Aké, L., Wilhelmi, M. R. (2012). Naturaleza del razonamiento algebraico elemental. Bolema, 42(B), 199-219, https://doi.org/10.1590/S0103-636X2012000200005

Godino, J.D., Neto, T. Wilhelmi, M.R., Aké, L., Etchegaray, S. \& Lasa, A. (2015). Niveles de algebrización de las prácticas matemáticas escolares. Articulación de las perspectivas ontosemiótica y antropológica. Avances de Investigación en Educación Matemática, 8, 117 - 142, https://doi.org/10.35763/aiem.v1i8.105 
Godino, J.D., Rivas, H., Arteaga, P., Lasa, A., Wilhelmi., M.R. (2014). Ingeniería didáctica basada en el EOS. Recherches en didactique des mathématiques, 34(23), 167-200, https://revue-rdm.com/2014/ingenieria-didactica-basada-en-el/

de Jong, T., van Joolingen, W.R. (2008). Model-Facilitated Learning. En J.M. Spector, M.D. Merrill, J. van Merriënboer y M.P. Driscoll (Eds.), Handbook of research on educational communications and technology (3a ed., pp. 457-468). New York: Lawrence Erlbaum.

Burke, M., Kennedy, P. (2011). GeoGebra: From simulation to formalization in teacher preparation and in-service programs. In L. Bu and R. Schoen (Eds.), Model-

Centered Learning (pp. 57-72). Rotterdam, NED: Sense Publishers.

Lasa, A. (2015). Jarduera matematikoa eredu dinamikoen laguntzaz [versión en vasco en el original: Actividad matemática con ayuda de modelos dinámicos]. Bilbo:

UEU, http://www.ueu.eus/denda/ikusi/jarduera_matematikoa_eredu_dinamikoen_lagun tzaz

Lasa, A. (2016). Instrumentación del medio material GeoGebra. Tesis doctoral. Pamplona: UPNA, http://academica-e.unavarra.es/handle/2454/20992

Lasa, A., Abaurrea, J., \& Belloso, N. (2016). Dynamic models for trigonometry. Revista do Instituto GeoGebra de São Paulo, 5(2), 30-55, http://revistas.pucsp.br/index.php/IGISP/article/view/28630

Lasa, A., Sáenz de Cabezón, A., Wilhelmi, M.R. (2009). Restrictions in the lecture of geometry with GeoGebra in primary school teacher education. Report of the First International GeoGebra Conference 2009, July 14th and 15th, at the RISC in Hagenberg near Linz, Austria.

Lasa, A., Wilhelmi, M.R. (2013a). GeoGebra en la formación de profesorado de ESO y de Bachillerato. Cónica 3, 30-32.

Lasa, A., Wilhelmi, M.R. (2013b). Use of GeoGebra in explorative, illustrative and demonstrative moments. Revista do Instituto GeoGebra de São Paulo, 2(1), $52-$ 64, http://revistas.pucsp.br/index.php/IGISP/article/view/15160

Lasa, A., Wilhelmi, M.R. (2015). Si Feynman hagués tingut GeoGebra. VII Jornades de l'Associació Catalana de GeoGebra, 20-21 de febrero, Universidad Pompeu Fabra, Barcelona.

Lasa, A., Wilhelmi, M.R., Belletich, O. (2014). A plot for Laika. Educação Matemática Pesquisa, 16(4), 1089-1110, http://revistas.pucsp.br/index.php/emp/article/view/22011/pdf 
Milrad, M., Spector, J.M., Davidsen, P.I. (2003). Model facilitated learning. En S. Naidu (Ed.), Learning and teaching with technology: Principles and practices (pp. 13-27). London: Kogan Page.

Trouche, L. (2000). La parabole du gaucher et de la casserole à bec verseur : étude des processus d'apprentissage dans un environnement de calculatrices symboliques. Educational Studies in Mathematics, 41, 239-264, https://hal.archives-ouvertes.fr/hal-01538672/

Wilhelmi, M.R. (2007). El momento del trabajo de la técnica en la evolución de un proceso de estudio: El caso de la determinación de una circunferencia. En L. RuizHigueras, A. Estepa, F.J. García (Ed.), Sociedad, escuela y matemáticas (pp. 177-197). Jaén: Universidad de Jaén. 